\title{
AutoEval mkll - Interaction Design for a VR Design Review System
}

\author{
Martin Naef, John Payne \\ Digital Design Studio \\ The Glasgow School of Art
}

\begin{abstract}
This paper summarizes the experience drawn from designing and revising a design review application prototype interface using immersive virtual reality technology and putting it into context with previous research in the field of 3D human-computer interaction. AutoEval was originally developed in collaboration with a major car manufacturer to enable intuitive analysis and manipulation of $3 \mathrm{D}$ models for users without a CAD or computer science background. This paper introduces the system and discusses the 3D interaction design decisions taken based on the observation and informal feedback of a large number of users.
\end{abstract}

Keywords: Virtual reality, multimodal interface, 3D interaction. Index Terms: I.3.6 [Computer Graphics] Methodology and Techniques - Interaction Techniques Additional

\section{INTRODUCTION AND MotIVATION}

The AutoEval 3D system for real-time visualization and interaction was originally developed to support advanced design for a major car company [1]. The project aimed to provide design review tools for executives, replacing the traditional clay models with virtual prototypes. The system was designed to provide an immersive visualization to enhance the understanding of form, but also provide basic interaction tools to quickly assess the impact of design changes such as moving or scaling components.

To support the design review process, the AutoEval system provides a selection of tools to visualize a virtual model, hands-on grab-and-move functionality to quickly investigate the design using a scene-in-hand paradigm, and various manipulator tools to move, rotate and scale the model or individual components. Various analysis tools, such as overlaying 2D drawings, slicing the model or moving light-sources have also been introduced.

Unlike traditional CAD systems, AutoEval was targeted towards novice or casual users such as corporate executives. As the project was clearly targeted at the concept stage, precision manipulations were not a priority. Designing a user interface for such a system therefore provided a set of challenges and required a very strong focus on intuitiveness and natural interaction.

This work in progress paper documents the basic interaction design and the experience gathered from combining a wide range of interaction paradigms into a single system. It presents the core object manipulation methods available to the user that will provide the basis for further usability evaluations. The experience gathered through the use of the AutoEval system confirms that many findings derived from focused experimental setups to test isolated aspects of 3D user interface design can be transferred successfully into a more application-oriented environment.

e-mail: m.naef@gsa.ac.uk

\section{RELATED WORK}

This work builds upon a wide range of findings in the field of human-computer interaction and 3D user interfaces in particular. While there has been a significant body of work for design review systems similar to AutoEval, this paper focuses on selected human-computer interaction aspects.

Research into workspace technology has been consistent since Ivan Sutherlands SketchPad Interface for drafting back in 1963. Recent work in the field of workspaces includes investigations into multi-modal and bimanual implementations to aid in the visualization of increasingly complex data sets and also the design of 3D geometry. DesignSpace [5] attempted to provide a collaborative $3 \mathrm{D}$ workspace for users to achieve real-time interaction with 3D geometry, but was limited by the technology available and mainly addressed high level theories in human factors. The Immersive Modeling Environment [15] attempts to merge the $2 \mathrm{D}$ and $3 \mathrm{D}$ workspace together by allowing designers to pull points off of the screen into the $3 \mathrm{D}$ space above to define shapes. Similarly, [11] combines $2 \mathrm{D}$ and $3 \mathrm{D}$ interaction in a collaborative setting. Many research institutions have approached the design of interactive workspaces. The responsive workbench [9] has been commercialized as the Fakespace Workbench. ERGODesk [6] introduces a workbench-like environment supporting both $2 \mathrm{D}$ and $3 \mathrm{D}$ interaction and a multimodal interface. These research projects typically focus on ergonomic and hardware aspects, whereas the work presented here focuses on interaction paradigms and user interface concepts.

[4] and [8] provide excellent in-depth reviews of the various aspects of $3 \mathrm{D}$ interface design. This review paper draws upon these findings and refers to them throughout the remainder of this paper, putting them into the context of an integrated application.

\section{USER INTERFACE DESIGN}

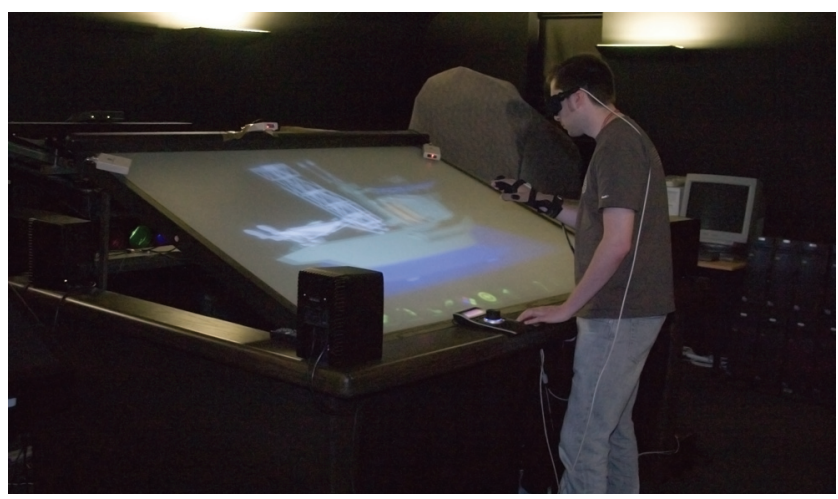

Figure 1. Hardware setup based on a Fakespace Workbench.

The AutoEval interface provides a multi-modal approach to human-computer interaction in 3D. It combines stereoscopic rendering with tactile and audio cues to ease spatial interaction 
with a virtual model. AutoEval mkII completely replaced the existing code base. Device control, configuration and visual and auditory rendering are now provided by a VR application programming interface, the blue-c API [12]. Even though the underlying VR toolkit supports a wide range of display and interaction devices in a transparent manner, the AutoEval mkII system is optimized for the Workbench environment (Figure 1).

The AutoEval system is targeted towards a user group consisting of non-experts without previous CAD or modeling experience. We could therefore design the interface free from constraints such as expectations or need to transfer existing skills from other modeling tools. Instead, we tried to bring intuitive paradigms derived from real-world experience into the virtual environment where possible.

\subsection{Glove Interface}

A tracked glove with bend sensors for all fingers (Immersion CyberTouch) worn on the right hand provides the main means of interaction with the virtual environment. Using the hand with finger flex gestures to point, select and pick objects provides a natural paradigm as it relates directly to how people interact with real objects.

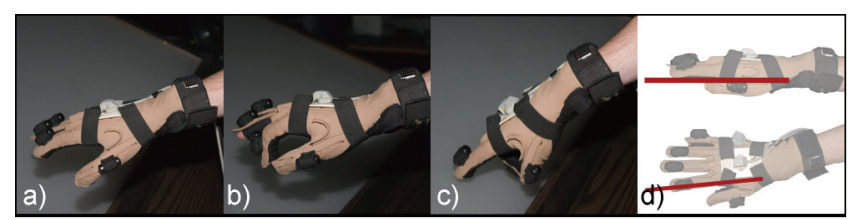

Figure 2. a) - c) Hand-gestures. d) Active segment.

The active area (intersection between the virtual scene and a ray) is aligned slightly below the index finger and parallel to the palm of the hand. It corresponds to a physical grasp or pinch gesture to pick up a small object. The AutoEval system supports a small set of hand gestures. The bend angles of thumb and index finger are compared against a threshold to distinguish between the relaxed state (Figure $2 \mathrm{a}$ ) and a pinch gesture (Figure $2 \mathrm{~b}$ ). A pointing gesture (Figure $2 \mathrm{c}$ ) is used to select menu options. Pointing is detected as an intersection between an active item and the selection segment (Figure $2 \mathrm{~d}$ ) when the hand is in the relaxed state (e.g. index finger is straight). The glove interface was transferred from the original AutoEval system without changes.

Compared to other 3D interaction devices, such as wands or 3D mice, the glove provides a very direct and intuitive interface as the user is "grabbing" things directly, instead of "pointing" at them through an additional indirection layer. Grabbing, however, only works with virtual objects that are scaled to fit the physical interaction volume, as opposed to pointing paradigms where objects can be far away. This interaction scale limitation was considered an acceptable trade-off in favor of intuitiveness, especially since stereoscopic depth perception becomes ineffective if objects are too far behind the projection plane.

Device acquisition can be an issue when using gloves [17]. Devices such as wands or 6DOF mice can easily be passed among collaborators to hand over the interaction. Sensor gloves on the other hand are somewhat cumbersome to pass. The single user nature of the workbench favors infrequent passing of control as it is required to pass both the main interaction device and the tracked glasses. Hence, the additional effort required putting on a glove was considered negligible.

\subsection{Direct Manipulation}

All editing modes can either be restricted to a single axis or parameter (e.g. translation along $\mathrm{Y}$ axis, uniform scale), or edit multiple parameters in parallel (e.g. free rotate/translate mode).
Axis restrictions are set explicitly through the menu system, whereas many $\mathrm{CAD}$ and $3 \mathrm{D}$ modeling packages use individual mouse buttons to select the active axis. For typical design review tasks, our users rarely desired axis restrictions; instead they usually preferred full $3 \mathrm{DOF}$ or $6 \mathrm{DOF}$ manipulations, as users tend to have difficulties separating rotations into components [13].

The system supports two distinct modes of manipulation. With direct manipulation mode, the user picks an object and manipulates it directly with the hand. Movement and rotation are applied without any scaling, although some edit modes restrict motion or rotation to a single axis. Picking the object and editing is done with a single pinch-move-release action sequence. This editing mode corresponds to the virtual hand metaphor as described and analyzed by Poupyrev et. al. [14]. Previous research is not fully conclusive about the effectiveness of the chosen virtual hand approach: Bowman and Hodges [3] state that some users preferred ray casting techniques for selection because they require the least effort and that naturalness is not necessarily a part of the requirement; Poupyrev's tests on the other hand favor the virtual hand for the positioning tasks. We found that for the non-expert target audience of AutoEval, naturalness is the most desirable property.

Our experience also confirms Zhai's [16] findings that the limited mobility of the wrists does impose limitations for the direct manipulation. AutoEval uses a 1:1 mapping of the wrist rotation to the object rotation, which is very natural, but necessitates several pick-rotate-release sequences to completely turn an object. The addition of indirect manipulation modes alleviates some of these limitations.

\subsection{Indirect Manipulation}

Indirect manipulation uses a disconnected user interface widget to apply a transformation to the last selected object. This manipulation mode involves a two step action sequence: first, the object is selected; then the edit mode is selected and applied by picking a user interface widget. The object selection is persistent, meaning various transformations can be applied in sequence without picking the object again. Indirect manipulation was only available for scaling using finger bend mode in the original AutoEval system.

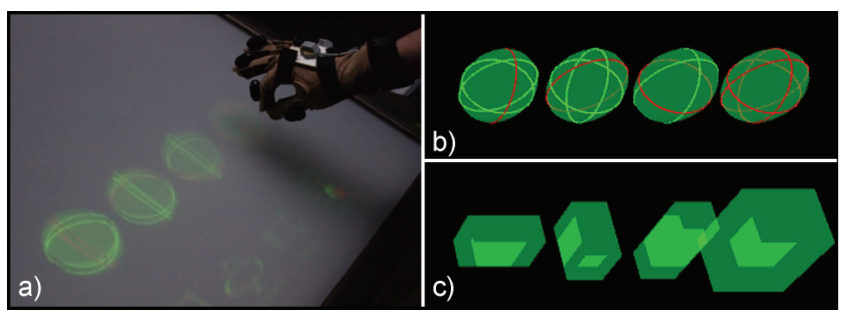

Figure 3. User interface widgets for indirect manipulation. a) Hand grabbing a rotation widget. b) and c) Screenshot of rotation and scaling widgets.

The indirect manipulation edit module supports three basic modes to define the transformation amount and direction. All modes described below apply a transformation (move, rotate, scale) along a single axis to the selected object. The edit is defined by the vector between the current hand position and where the associate UI widget was picked initially (Figure 3 ). The difference between the modes is in the mapping and scaling of the hand movement vector to the actual edit operation. Scaling is linear in all modes, but not 1:1 to work around the dexterity limitation of the hand and wrist.

The different modes described below were implemented for evaluation purposes: 
The wrist rotation mode provides a "virtual knob" paradigm to change a value; a gesture similar to somebody changing the volume on a stereo set. Rotation is always incremental, relative to the position where the widget was pinched. This mode enables precise edits since users have a fine and precise motor control for the wrists, but it is not always considered intuitive by users.

An additional 3DOF widget is supported to map wrist rotation onto object rotation along all axes.

Hand movement mode transforms the movement of the hand along the physical $\mathrm{x}$ axis into an edit amount. Unlike wrist rotation which is limited to roughly 180 degrees, hand movement allows a larger motion range, but provides slightly worse motor control as it requires movement of the whole arm or body if the natural limits of the lower arm range are exceeded [16].

Finger bend mode measures the average joint angle of the middle, ring and pinky finger using the glove sensors and transforms this difference into an edit value. This mode was introduced with the original AutoEval system for scaling objects. Finger bend mode is precise, but has a very limited range. We observed that repeated grab-bend-release gesture sequences to extend the edit range are awkward and tiresome for several reasons: the middle, ring and pinky fingers have to be moved to the start position before the pinch gesture is conducted, and there is simply no equivalent real-world gesture or action with such a strong separation between the index finger and the middle/ring/pinky group. In practice, it is also possible to change the direction of the edit after the pinch gesture, unless the fingers are held in a middle position, requiring a very conscious initiation of the hand gesture.

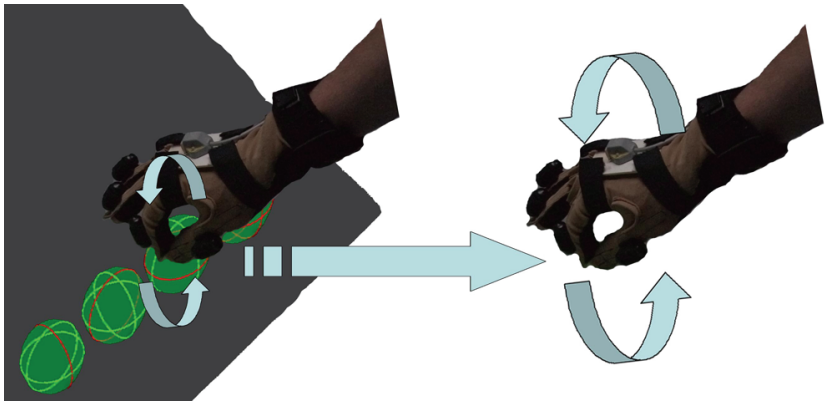

Figure 4. Mapping amplification control. The edit is amplified as the user moves the hand away from the widget after pinching.

Mapping Amplification Control: All of the moves above support an optional scaling modifier to enable a variable controldisplay ratio, enabling both very small scale, surgical edits as well as broad and fast transformations. The edit amount is amplified as the user moves the hand away along the physical $\mathrm{Y}$ axis from the edit widget after picking the widget. At the zero position (hand inside the widget), edits are scaled at roughly $1 / 5^{\text {th }}$ of the normal mode, at $20 \mathrm{~cm}$ distance they are equal to the default mode, and increased linearly as the hand moves away further. This mode is very useful for experienced users by allowing them to easily work around dexterity limits, but it requires explanation as it is not intuitive to first time users.

Indirect manipulation has several advantages over direct manipulation: the object no longer needs to reside within the physical working volume, the hand remains in a relatively relaxed position if the widgets are positioned sensibly, and the editing range can be scaled without breaking intuitiveness to enable precise edits. Direct manipulation, on the other hand, is significantly more intuitive and engaging than any of the indirect edit modes thanks to the direct link between the hand and the object. Both edit modes are available concurrently in the system under normal operation mode; it is the user's choice which mode is more appropriate for the task at hand.

\subsection{SpacePilot Interface}

The glove interface provides a very intuitive paradigm for broad manipulation in space at a 1:1 scale. It is less suitable for precise manipulations though, mostly because it relies on movements of the whole arm as opposed to the fingers, and because it lacks a tangible reference system and zero-point. An elastic device with very small movement, a 3Dconnexion SpacePilot that provides a small spring-loaded 6DOF "joystick" cap, was therefore added to the system. Unlike the glove-based manipulation methods where there is always a direct and 1:1 scale connection between the hand movement and the object, the SpacePilot defines a positional and rotational velocity: Pushing the cap moves the object into the respective direction until it is released, or rotates it if the cap is twisted. Using the SpacePilot in addition to the glove offers a few advantages: Using the left hand, the user can rotate, scale or move the selected object very precisely and conduct very smooth movements. Edit velocity can be kept very slow to enable precision edits since the glove interface already enables quick and broad manipulations.

The SpacePilot and its predecessors have been used extensively in desktop VR and modeling systems and as the main navigation device for projection-based immersive environments. Nonetheless, its use as a high-precision editing device to support and enhance a traditional editing system in the Workbench environment seems to be mostly ignored.

The workbench enables the user to explore the virtual object through head movement. Using a desktop device such as the SpacePilot, on the other hand, restricts the movement of the users, effectively grounding them to a fixed position. We found this not to be a problem because device acquisition and persistence is trivial - the user can simply let go of the device and grab it again later, separating the tasks of exploring and editing. In a collaborative setting, the device can also be passed quickly to another user.

The SpacePilot causes very little fatigue as the hand can comfortably rest on the device. On the downside, rate control is an acquired skill and there is little feedback from the device due to the very small movement range [17]. Novice users therefore tend to prefer the glove interface for most tasks.

Adding the SpacePilot extends the original AutoEval system towards a two-handed interaction paradigm where the right hand is used for menu and object selection and broad manipulation, whereas the left hand is used for precise edits. This setup deviates from Guiards's framework of bimanual asymmetric tasks [7]: In our case, the non-dominant hand controls the precise editing operations using the SpacePilot, whereas the right hand is used for large-scale edits and viewpoint manipulation using the scene-inhand paradigm. Although previous tests [2] suggest that using the dominant hand for precision edits would be more appropriate, the slow device acquisition of the glove as the main interaction device renders this approach impractical for our system. Precision for the non-dominant hand, however, is ensured at the cost of manipulation speed by keeping the transformation velocity low.

It is usually beneficial for precision editing operations to limit the active axes. The SpacePilot device includes an automatic dominant axis mode, which avoids manual selection of the edit axis through the menu system. This is considered the preferred mode, as full concurrent six degrees of freedom edits are significantly harder to control because there is only a subtle difference between moving the knob vs. tilting it. The axis separation difficulty is made worse by the fact that the user typically uses the non-dominant (left) hand to control the SpacePilot. Masliah [10] concludes that users tend to separate 
tasks into separate translation and rotation, which is facilitated by the dominant axis mode, although a separation into just 3D motion or 3D rotation would be more appropriate given people's difficulty to decompose the rotation [13].

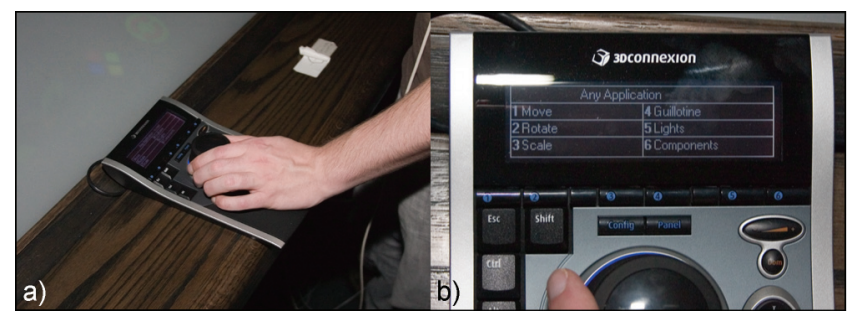

Figure 5. a) 3Dconnexion SpacePilot. b) Custom menu shortcuts.

The SpacePilot buttons were programmed to provide shortcuts to frequently used menu options, although it is usually faster to access the menu with the glove worn on the dominant hand. The lower border of the Fakespace Workbench provides just enough space to support the device at a comfortable height for the majority of users (Figure 5).

\section{Discussion AND FUtURE WORK}

The original AutoEval system and its mkII successor have been used as technology showcases for several years. The application was demonstrated to hundreds of visitors from a very wide background, including school children, students, researchers and professionals in design, engineering, medicine, education, advertisement, communication, and management. The vast majority of users enjoyed using the system, with many of them being very enthusiastic about the opportunities opened by this kind of environment.

Analyzing the reactions with more scrutiny, it is difficult to distinguish without a formal assessment between the immediate "wow"-effect induced by experiencing a VR system hands-on for the first time vs. judging the actual success of the design against set criteria. Nonetheless, observing such a large number of users in an informal setting provided a good insight into how quickly people pick up different interaction methods in an immersive 3D workspace environment.

The revised AutoEval version is still under constant development as a test bed for interaction and interface ideas. Formal user tests including task performance measurements and questionnaires are in process to validate the design decisions and finalize the selection of indirect manipulation metaphors.

\section{ACKNOWLEDGEMENTS}

Thanks to ETH Zurich for providing the blue-c API, and the developers of the original AutoEval system for inspiration of the user interface paradigm. Additional thanks to Vassilis Charissis for providing 3D models and graphic design.

\section{REFERENCES}

[1] Anderson, P., Kenny, T., Ibrahim, S. 2002. "The role of emerging visualisation technologies in delivering competitive market advantage." 2nd International Conference on Total Vehicle Technology, Institute of Mechanical Engineers, University of Sussex, Brighton, UK, pp. 87-97.

[2] Balakrishnan, R. and Kurtenbach, G. 1999. "Exploring bimanual camera control and object manipulation in 3D graphics interfaces." In Proceedings of the SIGCHI Conference on Human Factors in Computing Systems: the CHI Is the Limit (Pittsburgh, Pennsylvania, United States, May 15 - 20, 1999). CHI '99. ACM Press, New York, NY, 56-62.
[3] Bowman, D. A. and Hodges, L. F. 1997. "An evaluation of techniques for grabbing and manipulating remote objects in immersive virtual environments." In Proceedings of the 1997 Symposium on interactive 3D Graphics (Providence, Rhode Island, United States, April 27 - 30, 1997). SI3D '97. ACM Press, New York, NY, 35-ff.

[4] Bowman, D.A., Kruijff, E., LaViola, J.J., Poupyrev, I. 2005. 3D User Interfaces - Theory and Practice. Addison-Wesley, ISBN 0-20175867-9

[5] Chapin, W. L., Lacey, T. A., and Leifer, L. 1994. "DesignSpace: a manual interaction environment for computer-aided design." In Conference Companion on Human Factors in Computing Systems (Boston, Massachusetts, United States, April 24 - 28, 1994). C. Plaisant, Ed. CHI '94. ACM Press, New York, NY, 47-48.

[6] Forsberg, A., LaViola J., and Zeleznik, R. 1998. "ErgoDesk: A Framework for Two and Three Dimensional Interaction at the ActiveDesk." In Proceedings of the Second International Immersive Projection Technology Workshop, Ames, Iowa, May 11-12.

[7] Guiard, Y., 1987. "Asymmetric Division of Labor in Human Skilled Bimanual Action: The Kinematic Chain as a Model", In Journal of Motor Behavior, Vol. 19(4), pp. 486-517

[8] Hinkley, K., Pausch, R., Goble, J., Kassell, N., 1994. "A survey of design issues in spatial input." In UIST '94: Proc. ACM Symposium User Interface Software Technology 1994, Marina Del Rey, CA, pp. 213-222.

[9] Krueger, W.; Bohn, C.-A.; Froehlich, B.; Schueth, H.; Strauss, W.; Wesche, G., 1995. "The Responsive Workbench: a virtual work environment," Computer, vol.28, no.7, pp.42-48, IEEE, Jul. 1995

[10] Masliah, M. R. and Milgram, P. 2000. "Measuring the allocation of control in a 6 degree-of-freedom docking experiment." In Proceedings of the SIGCHI Conference on Human Factors in Computing Systems. The Hague, The Netherlands, April 01 - 06, 2000. CHI '00. ACM Press, New York, NY, 25-32.

[11] Nakashima, K., Machida, T., Kiyokawa, K., and Takemura, H. 2005. "A 2D-3D integrated environment for cooperative work." In Proceedings of the ACM Symposium on Virtual Reality Software and Technology (Monterey, CA, USA, November 07 - 09, 2005). VRST '05. ACM Press, New York, NY, 16-22.

[12] Naef, M., Staadt, O., Gross, M., 2004. "blue-c API: A Multimedia and 3D Video Enhanced Toolkit for Collaborative VR and Telepresence." Proceedings of ACM SIGGRAPH International Conference on Virtual-Reality Continuum and its Applications in Industry 2004. VRCAI 2004, Singapore, June 16-18.

[13] Parsons, L. 1995. "Inability to reason about an object's orientation using an axis and angle of rotation", Journal of Experimental Psychology: Human Perception and Performance, 21(6), 1995, pp. 1259-1277

[14] Poupyrev, I., et al. 1998. "Egocentric object manipulation in virtual environments: empirical evaluation of interaction techniques". Computer Graphics Forum, EUROGRAPHICS'98 issue. 17(3): pp. 41-52.

[15] Yoshimori, H., 2000. "Combination of Two- and Three-Dimensional Space for Solid Modeling", SIGGRAPH conference Sketches \& Applications, ACM, Los Angeles, California.

[16] Zhai, S., Milgram, P., and Buxton, W. 1996. The influence of muscle groups on performance of multiple degree-of-freedom input. In Proceedings of the SIGCHI Conference on Human Factors in Computing Systems: Common Ground (Vancouver, British Columbia, Canada, April 13 - 18, 1996). CHI '96. ACM Press, New York, NY, 308-315.

[17] Zhai S., 1998. "User performance in relation to 3D input device design", Journal of Computer Graphics, Vol. 32(4), 1998, ACM Press, New York, US, pp. 50-54. 